\title{
e-Migrinter
}

$20 \mid 2020$

Hospitalité et migration

\section{Réseaux des villes hospitalières : un panorama global}

\section{Thomas Lacroix}

\section{CpenEdition}

Journals

Édition électronique

URL : https://journals.openedition.org/e-migrinter/2281

DOI : 10.4000/e-migrinter.2281

ISSN : 1961-9685

Éditeur

UMR 7301 - Migrinter

Référence électronique

Thomas Lacroix, "Réseaux des villes hospitalières : un panorama global », e-Migrinter [En ligne], 20 | 2020, mis en ligne le 23 juillet 2020, consulté le 20 mai 2021. URL : http://journals.openedition.org/emigrinter/2281 ; DOI : https://doi.org/10.4000/e-migrinter.2281

Ce document a été généré automatiquement le 20 mai 2021.

Tous droits réservés 


\title{
Réseaux des villes hospitalières : un panorama global
}

\author{
Thomas Lacroix
}

1 Il existe un certain parallélisme entre les développements politiques à l'échelle mondiale en matière de changement climatique et de migration. À la sclérose de la coopération intergouvernementale, répond le dynamisme des villes et autres acteurs locaux. Les organisations internationales voient dans ces nouveaux acteurs des partenaires sur lesquels elles peuvent s'appuyer pour contourner la résistance des États. La recherche sur les villes dans la coopération internationale a principalement porté sur le changement climatique (Bulkeley et al., 2003; Kern et Bulkeley, 2009; Fünfgeld, 2015). Mais une dynamique similaire se dessine dans le domaine des migrations internationales. Les réseaux de villes sur les questions migratoires se sont multipliés au cours de ces dernières années : villes sanctuaires en Grande Bretagne et aux États-Unis, l'Association Nationale des Villes et Territoires Accueillants en France, etc. Dans le même temps, des organisations européennes et internationales, au premier rang desquelles l'OIM (Organisation internationale pour les migrations), ont manifesté un intérêt croissant pour leurs activités (Organisation internationale pour les migrations 2015 ; Forum économique mondial 2017).

2 Cet article a pour objectif de donner un aperçu d'une dynamique émergente, mais qui s'inscrit dans l'établissement d'une gouvernance des migrations internationales (Lacroix et Desille 2018) : quels sont les facteurs permettant d'expliquer l'essor des réseaux de ville en matière migratoire? Quelles sont les différentes catégories de réseaux et que font-ils ? Comment ce dynamisme s'inscrit-il dans la mise en place d'une gouvernance globale de la migration et de l'intégration? Afin de répondre à ces questions, j'aborderai successivement les conditions d'émergence de ces réseaux de villes, puis je présenterai une typologie des différents réseaux. Je tenterai de définir ce qu'est un "réseau de ville ", exercice difficile tant les regroupements de municipalités peuvent prendre une grande diversité de formes. La conclusion évoquera brièvement les initiatives prises par l'oIM et les agences onusiennes pour coopter ces acteurs au sein de la gouvernance mondiale des migrations. 


\section{Les conditions d'émergence de réseaux de villes hospitalières}

\section{Un bref aperçu historique}

3 L'histoire des réseaux de villes remonte au moins au XIXe siècle (Saunier et Ewen, 2008), mais la présence de la question migratoire sur leur agenda est relativement récente. Cette introduction date des années 1980, en réaction aux politiques d'intégration et d'asile existantes. Au Royaume-Uni, le Greater London Council (GLC), et les boroughs ${ }^{1}$ travaillistes de Londres (Lambeth, Brent, Hackney et Haringey), mais aussi Manchester, Birmingham ou Bradford ont promu une politique de relations ethniques proactive. En témoigne l'inclusion, en 1983, d'une clause obligeant les entreprises privées sous contrat avec la municipalité d'obéir aux régulations en matière d'égalité de traitement (equal oportunity clause), (Solomos, 2003: 108). Cette mesure, en confrontation directe avec la ligne politique du gouvernement Thatcher a précipité la dissolution du GLC en 1986. Aux États-Unis, en 1985, San Francisco s'est déclarée « ville sanctuaire » contre la politique restrictive à l'égard des réfugiés d'Amérique centrale (Ridgley, 2008). Le mouvement revendique maintenant plus de 500 membres.

Dans les années 1990, des réseaux de villes à l'échelle européenne se sont formés parallèlement à la construction d'un espace politique européen. La Commission européenne a largement soutenu ce processus, avec l'intention de développer des canaux de communication directs avec les autorités locales. Le Conseil des Communes et Régions d'Europe (CCRE) est devenu leur représentant à Bruxelles, aux côtés de l'Assemblée des Régions européennes (Hooghe, 1995). D'autres réseaux tels qu'Eurocités sont devenus des partenaires de la Commission européenne dans la mise en œuvre des politiques d'intégration des migrants (Flamant, 2014). Eurocités, qui regroupe aujourd'hui 190 villes réparties dans 39 pays, est initialement un réseau de ville créé au début des années 1990 pour porter la voix de grandes villes européenne « secondaires » (qui ne sont pas des capitales) telles que Barcelone, Lyon ou Birmingham. Il fut progressivement inscrit dans le paysage institutionnel européen en tant que partenaire régulier des institutions de l'Union européenne. Si la période des années 1980 était celle de l'émergence de réseaux nationaux et politisés par des gouvernements locaux de gauche en confrontation avec leurs gouvernements positionnés à droite, les réseaux européens créés dans les années 1990 s'insèrent dans le paysage institutionnel de l'Union en tant que partenaires techniques.

5 Longtemps, le nombre de villes véritablement impliquées est resté limité à un petit nombre de grandes villes de gauche, désireuses de développer leur propre agenda international : Rotterdam, Anvers, Birmingham, Lyon, Barcelone... Cette dernière ville en est un bon exemple. Barcelone est un membre fondateur d'Eurocités, elle accueille par ailleurs le siège du Conseil des Cités et Gouvernements Locaux Unis et elle est membre fondateur du Forum pour la mobilité humaine et le développement. Barcelone, comme Lyon ou Anvers et d'autres métropoles secondaires ont une présence sur la scène internationale motivée par le souhait de développer une identité qui se distingue de celle des capitales. L'implication de Barcelone doit être comprise dans le contexte particulier du régionalisme espagnol et de la relation de la ville avec Madrid et la Catalogne. En revanche, les autorités locales françaises, hormis quelques exceptions 
(Lyon, Nantes) ont été notoirement absentes de cette arène, à la fois pour des raisons techniques (manque de maîtrise de l'anglais) et idéologiques (antagonisme entre les approches multiculturalistes et républicaines).

\section{Les motifs de l'expansion récente}

6 À la fin des années 1990 et au début des années 2000, débute une nouvelle période caractérisée par une expansion mondiale de la formation de réseaux de villes investis sur les questions migratoires. Cette évolution a été déclenchée par deux paradoxes auxquels les autorités locales sont confrontées.

7 Un premier paradoxe est inscrit dans les politiques de décentralisation qui attribuent plus de responsabilités aux autorités locales, notamment en matière de développement local, mais avec moins de capacités financières pour le faire. Il faut replacer cette évolution dans le contexte des années 1990, période marquée par une vague de décentralisation dans le monde : la majorité des États du monde ont adopté des lois qui élargissent les pouvoirs des autorités locales dans le but de favoriser les partenariats avec les sphères associatives et économiques locales (Ivanyna et Shah, 2012; Manor, 2004). Mais cela va généralement de pair avec une réduction de leurs ressources financières. Ce type de politique a d'abord été mis en œuvre dans les pays du Sud et va souvent avec la croissance d'un secteur associatif axé sur le développement local (Gazzotti, 2018). Dans les pays du Nord, la politique de décentralisation est associée avec une stratégie d'allègement des responsabilités de l'État central et de compression des finances publiques. Nous avons un exemple récent au Royaume-Uni, où la loi sur le localisme de 2011 et la loi de 2014 sur les villes et les gouvernements locaux a élargi leurs capacités juridiques, notamment dans le domaine économique, mais a réduit de $40 \%$ le financement public octroyé aux municipalités, une réduction qui doit atteindre $100 \%$ en 2020 .

8 Contrairement à la génération précédente attachée à réformer l'appareil d'État pour s'engager sur le marché international, ces politiques de décentralisation ont pour but d'insérer les autorités locales dans un environnement concurrentiel dans lequel divers acteurs publics et privés coopèrent et / ou sont en concurrence pour des ressources économiques rares. Les acteurs publics locaux sont censés compenser la perte de ressources publiques en valorisant leurs avantages comparatifs et en tirant parti de leurs liens avec les acteurs locaux, nationaux et internationaux. Dans les principaux pays d'émigration, l'engagement croissant des municipalités vis-à-vis de leurs expatriés et la multiplication des projets d'investissement et / ou de développement soutenus par des émigrants sont le résultat de cette politique. Les politiques de décentralisation en Afrique du Nord, en Asie ou en Amérique latine sont à comprendre en lien avec l'essor des politiques liant migration et développement au cours de la même période. Dans les pays d'immigration, les politiques d'intégration considérant les migrants et l'immigration comme un moteur pour l'entrepreneuriat local sont également un produit de ce nouvel élan néolibéral. Un exemple est celui de l'approche de la régénération urbaine proposée par Richard Florida (Florida, 2014). Particulièrement influente aux États-Unis, cette conception appuie le soutien d'une classe "créative » dans les quartiers gentrifiés rassemblant migrants, artistes, communautés gays, étudiants ou jeunes entrepreneurs. Ces quartiers produisent une image plus jeune et positive de la ville tout en favorisant le développement économique. En Europe, les 
«politiques de la diversité » visant à valoriser l'image de la ville se sont répandues dans des villes d'Europe du Nord telles que Vienne ou Amsterdam (Hoekstra, Kohlbacher et Rauhut, 2018).

9 Mais les municipalités du Nord sont confrontées à un deuxième type de paradoxe, relatif aux politiques migratoires. D'une part, on observe un «tournant local» des politiques d'intégration: les villes sont reconnues comme un niveau clé de la mise en œuvre des stratégies de gestion des populations immigrées. Depuis le milieu des années 2000, l'Union européenne encourage la mise en œuvre de politiques municipales d'intégration (financement d'associations de soutiens à l'insertion, création de services administratifs dédiés, mise en place de formation en langue, etc.). Mais, parallèlement, elles doivent faire face aux effets de politiques migratoires plus strictes, qui renforcent la précarité des populations immigrées et compromettent leur capacité à s'intégrer dans le tissu socio-économique local. Une fois encore, le Royaume-Uni l'illustre : en 2012, la réforme de la politique d'intégration visait à «créer les conditions de l'intégration ", mais en 2014, la loi sur l'immigration a eu pour objectif de créer " un environnement hostile " pour les migrants sans titre de séjour valide (Hiam, Steele, et McKee, 2018). De manière générale, les autorités locales sont touchées par une politique "d'internalisation» du contrôle des frontières qui délègue de facto à une diversité d'acteurs publics et privés des responsabilités en matière de contrôle des populations immigrées et de la régularité de leur séjour. On pense par exemple aux demandes faites par l'administration centrale (en France, la préfecture) de mobiliser les services de police municipaux pour l'évacuation de squats et campements informels ou d'appréhender les personnes en situation irrégulière. Dans la section suivante, je reviendrai sur la façon dont la « crise migratoire » (expression qualifiant ici le désordre politique suscité par la vague d'exilés en provenance d'Afrique et du Moyen Orient) de 2015-2016 a accentué la tension entre ces politiques de migration et d'intégration et a accéléré la formation de ces réseaux de villes.

10 Les nouvelles approches d'inspiration néolibérale du développement et de la régénération urbaine mais aussi la (dés-) articulation des politiques d'intégration et de migration, ont contribué à l'émergence de réseaux de villes. Ces dynamiques ont généré des contradictions auxquelles les autorités locales ont cherché à répondre collectivement en partageant leurs expériences. La section suivante décrit les types de réseaux existants et leurs activités respectives.

\section{Une typologie des réseaux de villes hospitalières}

11 Cette section s'appuie sur la constitution et l'analyse d'un échantillon de 50 réseaux de villes identifiés dans le monde. L'échantillon a été élaboré à partir de recherches sur internet et de bases de données réalisées par différentes organisations (le programme Migration4development, la ville de Montréal). La définition même de ce qu'est un réseau de ville est une affaire plus fastidieuse qu'il n'y paraît. Initialement, les recherches sur les réseaux de ville sont des recherches sur les villes mises en réseaux par différents flux (flux d'argent, d'information, réseaux commerciaux et financiers, etc.). On pense ici aux travaux sur les réseaux de villes globales ou sur les réseaux qui connectent les villes avec leur "hinterland» (Taylor et Derudder, 2004). Nous nous intéressons ici à des collectifs de municipalités formés autour de besoins communs. Les travaux sur les réseaux de ville, liés aux questions migratoires ou pas, se contentent 
généralement de proposer une définition assez vague de ce type d'organisation. Nous définissons un réseau de ville comme un regroupement de municipalités doté d'une structure organisationnelle (un bureau, un comité de liaison, une charte, etc.) et engagés autour d'objectifs communs. Il convient de préciser les éléments de cette définition. On peut distinguer deux cas de figures : les réseaux qui se forment en vue de se mobiliser collectivement autour d'objectifs communs et les réseaux qui sont le résultat de mobilisations collectives. Le premier cas recouvre les associations et autres institutions qui structurent les collaborations interurbaines (comme par exemple Eurocities en Europe ou la Fédération des Municipalités canadiennes). Les objectifs qu'elles poursuivent peuvent être précis (développer une politique d'intégration) ou généraux (assister ses membres dans différents domaines ou promouvoir une diplomatie urbaine à l'échelle internationale). Les membres peuvent être exclusivement composés de municipalités (soit des élus, soit du personnel administratif et technique), ou être des regroupements composites incluant également des ONG, des représentants ministériels ou des organisations internationales. Ils peuvent être composés d'un groupe stable de membres, mais la plupart des réseaux sont en fait des regroupements dont la composition fluctue au gré des stratégies de chacun et du calendrier électoral (lorsqu'une ville se retire d'un réseau suite à un changement de majorité). C'est particulièrement vrai pour une forme spécifique de réseau de villes que sont les forums représentatifs des collectivités territoriales auprès des organisations internationales : le Congrès Permanent des Pouvoirs locaux et Régionaux auprès du Conseil de l'Europe ou le Forum des Maires pour la mobilité, la migration et le développement qui incarne la voix des municipalités auprès de l'Organisation des Nations Unies ou de l'Organisation Internationale pour les Migrations. La structure institutionnelle peut être centralisée avec un siège administratif dédié, ou au contraire totalement décentralisée, chaque municipalité gérant sa propre implication (par exemple les réseaux formés autour d'une charte ou d'un appel commun comme Sello Migrante, qui regroupe des municipalités chiliennes engagées dans l'accueil des réfugiés haïtiens). On le voit, les réseaux constitués en amont d'une mobilisation collective peuvent prendre des formes très différentes. À ceux-là s'ajoutent les réseaux formés en aval d'une mobilisation: les réseaux constitués autour d'un projet ou une campagne d'information. Un exemple est celui de City-2-city, un projet d'échange d'expériences entre villes des deux côtés de la Méditerranée piloté par l'agence ICMPD ou encore le projet «Empowering Refugee Hosting Districts» de l'Uganda Local Government Association. Un autre est celui des Communes hospitalières en Belgique, issu d'une campagne de mobilisation autour de l'accueil et de la perception des migrations lancée par l'ONG CNCD-11.11.11. Dans les deux cas, le réseau s'est formé à la suite de l'intégration des communes dans ces mobilisations. Le tableau ci-dessous distingue 4 grandes catégories en fonction de leur échelle (nationale / internationale) et de leur place dans le champ des réseaux municipaux (spontanés / cooptés). Les réseaux cooptés sont des réseaux qui ont été créés par des États et/ou des organisations internationales, le plus souvent pour représenter les collectivités territoriales. À l'inverse, les réseaux spontanés sont des regroupements créés par les collectivités territoriales elles-mêmes pour porter une voix des municipalités sur les scènes nationales ou internationales. Une subdivision distingue les groupements spécialisés ou généralistes. Les réseaux spécialisés ne s'occupent que des questions migratoires, les généralistes ont un panel d'activités plus large. 


\begin{tabular}{|c|c|c|}
\hline & Réseaux spontanés & Réseaux cooptés \\
\hline $\begin{array}{l}\text { Échelle } \\
\text { nationale }\end{array}$ & $\begin{array}{l}\text { Réseaux contestataires: ANVITA, Cities of } \\
\text { sanctuary UK, Rete di Comuni Solidali, } \\
\text { Sanctuary Cities US, Sanctuary Cities } \\
\text { Canada, Welcoming cities (Australie), } \\
\text { Welcoming Communities (Nouvelle } \\
\text { Zélande), Welcoming cities (US), Comité des } \\
\text { villes avec une concentration de population } \\
\text { étrangère (Japon), Cities for Action, } \\
\text { Communes hospitalières, Inclusive Cities, } \\
\text { Sello Migrante, Solidarity cities }\end{array}$ & $\begin{array}{l}\text { Associations nationales: Cités Unies } \\
\text { France, Cités Unies Liban, Fédération des } \\
\text { Municipalités Canadiennes, Local } \\
\text { Government Association (UK), South African } \\
\text { local government association, Vereniging } \\
\text { van Nederlandse Gemeenten (VNG), Uganda } \\
\text { Local government association, Diverty } \\
\text { Netzwerk, National League for cities }\end{array}$ \\
\hline $\begin{array}{l}\text { Échelle } \\
\text { transnationale }\end{array}$ & $\begin{array}{l}\text { Réseaux généralistes: Eurocities, Global } \\
\text { Parliament of Mayors, Fearless cities } \\
\text { Réseaux spécialisés transnationaux: } \\
\text { International Cities of Refuge Network, } \\
\text { Observatoire des Maires sur le Vivre } \\
\text { ensemble, C-Mise, Welcoming International }\end{array}$ & $\begin{array}{l}\text { Réseaux généralistes: city alliance, CEMR, } \\
\text { Cités des gouvernements locaux unis (CGLU), } \\
\text { Congrès Permanent des Pouvoirs Locaux et } \\
\text { Régionaux, Fédération latino-américaine des } \\
\text { villes, municipalités et associations de } \\
\text { pouvoirs locaux, Mercociudades, Metropolis, } \\
\text { Urbact, Association Internationale des } \\
\text { Maires Francophones, Euro-Mediterranean } \\
\text { Regional and Local Assembly (ARLEM) } \\
\text { Réseaux spécialisés: Coalition } \\
\text { Internationale des Villes contre le Racisme } \\
\text { (ICCAR), Intercultural Cities Network, } \\
\text { International Coalition of Inclusive and } \\
\text { Sustainable Cities, Mayoral Forum on } \\
\text { Human Mobility and Development, } \\
\text { Observatoire des villes inclusives, City2city, } \\
\text { European network of cities for local } \\
\text { integration policies for migrants (CLIP), } \\
\text { Ciudad Solidaria, Integrating cities, Host } \\
\text { Municipalities Learning Network }\end{array}$ \\
\hline
\end{tabular}

\section{Réseaux nationaux}

Une première catégorie rassemble les réseaux créés à l'échelle nationale. Il faut ici distinguer les réseaux contestataires initiés en réaction aux politiques migratoires dans les États d'accueil et les associations de collectivités territoriales à vocation généraliste.

\section{Les associations municipales nationales}

13 Les associations nationales constituent à la fois la voix officielle des communes auprès de l'État et l'échelon de base de l'édifice international des réseaux municipaux chapeauté au niveau onusien par Cités et Gouvernements Locaux Unis (CGLU). Ce type d'organisation, à l'instar de l'Association des Maires de France, existe dans tous les pays du monde. Mais toutes ne sont pas actives sur la question des migrations. Le tableau cidessus inclut un échantillon d'associations dont l'investissement dans ce domaine est avéré. C'est le cas de l'association néerlandaise "Vereniging van Nederlandse Gemeenten » 
qui a réalisé un système d'échange de bonnes pratiques en matière d'intégration. Ce type d'implication n'est pas spécifique aux pays du Nord. L'Uganda local government association a mis en place le projet «Empowering Refugee Hosting Districts » dans trois districts hébergeant des camps de réfugiés. De même, la South African local government association, intègre un groupe de travail sur les migrations depuis les années 2009/2010.

Une autre catégorie d'organisations de portée nationale centrées sur les questions migratoires regroupe les associations affiliées à la Fédération Mondiale des Cités Unies qui promeut les jumelages. Par exemple, Cités Unies France a soutenu une vingtaine de projets interurbains portant sur les questions migratoires.

\section{Les réseaux contestataires}

Les organisations représentatives présentées ci-dessus sont des organisations "généralistes" dont une minorité s'intéresse aux migrations. Par contraste, les réseaux nationaux focalisés sur cette question sont généralement créés en réaction aux politiques de l'État à l'égard des immigrés. Cette catégorie regroupe les mouvements des villes sanctuaires aux États-Unis et au Royaume-Uni, l'association nationale des villes et territoires accueillants (ANVITA) en France ou encore l'association «RECOSOL» en Italie. Comme mentionné ci-dessus, le mouvement des villes sanctuaires s'inscrit dans une longue histoire de confrontations entre le niveau local et l'État central. Dans ce contexte, les autorités locales peuvent adopter des mesures en contradiction avec la politique de l'État. Un exemple est la délivrance de cartes d'identité municipales à des sans-papiers aux États-Unis ou encore l'instruction donnée au personnel municipal (y compris la police) de ne pas demander leurs papiers aux immigrants. Le mouvement Welcoming Cities est initialement une organisation américaine qui s'appuie sur le mouvement des villes sanctuaires, mais qui comprend également des ONG. Des organisations similaires sont en cours de création en Australie, en Nouvelle-Zélande et à Berlin. Il a pour objectif à faciliter la collaboration entre les autorités locales et les organisations de la société civile afin de soutenir une gestion concertée des programmes d'accueil et d'intégration. Une partie de leurs activités s'adresse également au grand public et aux décideurs par le biais de campagnes de sensibilisation.

La situation est différente en France où le système centralisé laisse moins de marge de manœuvre aux collectivités territoriales : leurs compétences en matière migratoire est tributaire du contrôle préfectoral, notamment dans les domaines de la sécurité et de l'administration des titres de séjour. L'implication des municipalités sur la question de l'intégration remonte au début des années 1980 (Flamant, 2014). Cette implication a été portée par quelques grandes villes telles que Lille, Nantes, Strasbourg ou Lyon autour des questions d'intégration. Mais ce n'est qu'au moment de «la crise » de 2015 qu'un effort coordonné visant à peser sur les politiques de l'État a pris forme. La décision d'ouvrir un camp humanitaire dans la ville de Grande-Synthe a constitué un tournant. Le maire de Grande-Synthe a choisi d'ouvrir un camp d'accueil pour immigrants de transit répondant aux normes du HCR en mars 2016. Un deuxième camp a été ouvert à Paris quelques mois plus tard (même si celui-ci a été critiqué pour servir de fins d'ordre plus sécuritaire qu'humanitaire). L'objectif était non seulement de répondre aux besoins urgents des immigrés, mais également de proposer un contre-modèle humanitaire à la gestion sécuritaire qui prévalait à Calais. Ces camps ont cristallisé l'attention médiatique: c'était la première fois que des maires prenaient une telle 
position à l'encontre des politiques d'immigration actuelles. La deuxième étape a débuté après le démantèlement de la « jungle » de Calais en octobre 2016. La dissolution du camp a été suivie par la réinstallation de la population immigrée dans d'autres régions de France, y compris dans des villes et villages plus petits non concernés jusque-là par ces flux. Cela a déclenché une demande accrue de ressources, de compétences et de savoir-faire de la part des associations et des autorités locales en charge de cet accueil. L'accueil des immigrés s'est fait sur une base volontaire: de nombreuses collectivités territoriales (et leur population) étaient réellement disposées à accueillir des immigrants. Enfin, en décembre 2017, une pétition ${ }^{2}$ a été publiée dans le journal Le Monde, signée par les maires de grandes villes de France (notamment Bordeaux, Lille et Strasbourg). La pétition demandait un soutien financier accru pour permettre aux autorités locales de répondre aux besoins d'immigrants vulnérables en hiver. Mais le texte demandait également de relâcher la pression policière sur les immigrants. La pétition était signée par des maires appartenant à la droite comme à la gauche. Elle a révélé que l'opposition à la politique de l'État ne suivait pas le clivage droite / gauche.

17 Ces mobilisations ont préfiguré la création de l'Association Nationale des Villes et Territoires Accueillants (l'ANVITA) en septembre 2018. Un groupe de dirigeants municipaux membres du parti écologiste dirigé par Damien Carême, maire de GrandeSynthe, est au centre de cette initiative. Au-delà de cette commune, les membres fondateurs sont Grenoble (vert), le 1er arrondissement de Lyon (PS), Ivry-sur-Seine (communiste), Montreuil (communiste), Briançon (PS), Nantes (PS), Strasbourg (PS), St Denis (communiste). L'objectif du réseau est de promouvoir une politique et des pratiques articulées autour de l'idée de l'accueil inconditionnel.

18 Cet accent mis sur l'accueil à court terme des personnes venant d'arrivée (ou en transit vers d'autres destinations) plutôt que sur l'intégration à plus long terme, est commun à nombre de réseaux contestataires (nous l'avons également trouvé dans le nom "Welcoming America» ou "Welcome International »). Il est typique d'un contexte où les trajectoires migratoires sont de plus en plus instables et temporaires et donc où les problématiques d'accueil prennent le pas sur celle d'intégration. Leurs activités comprennent le lobbying des décideurs politiques nationaux, mais également la création de liens avec des ONG et des chercheurs (cf. le partenariat avec le nouvel institut Convergence Migration dans le cas de l'ANVITA, ou encore les liens entre Welcoming America et le programme Inclusive Cities de l'Université d'Oxford).

Ce rapide aperçu des réseaux "contestataires » sur les migrations montre qu'ils ont tendance à être hautement politisés et spécialisés : ils sont créés à dessein pour traiter les problèmes d'immigration en réaction aux politiques nationales. L'exemple français montre comment le contexte de "crise» de 2015 a transformé cette tendance. En premier lieu, le contexte d'urgence a incité les villes à s'impliquer dans des réseaux plus larges, moins pour des raisons idéologiques que pour des raisons pragmatiques: les besoins immédiats d'assistance et de conseil ont accéléré le processus de mise en réseau. La situation a élargi l'engagement des villes au-delà du noyau d'acteurs ayant manifesté leur intérêt pour les questions de migration et d'intégration pour des raisons idéologiques. En second lieu, ces nouveaux réseaux expriment maintenant publiquement leurs préoccupations non seulement sur les questions d'intégration, mais aussi sur les politiques d'asile. 
Carte 1 : Trois réseaux de villes contestataires en Europe

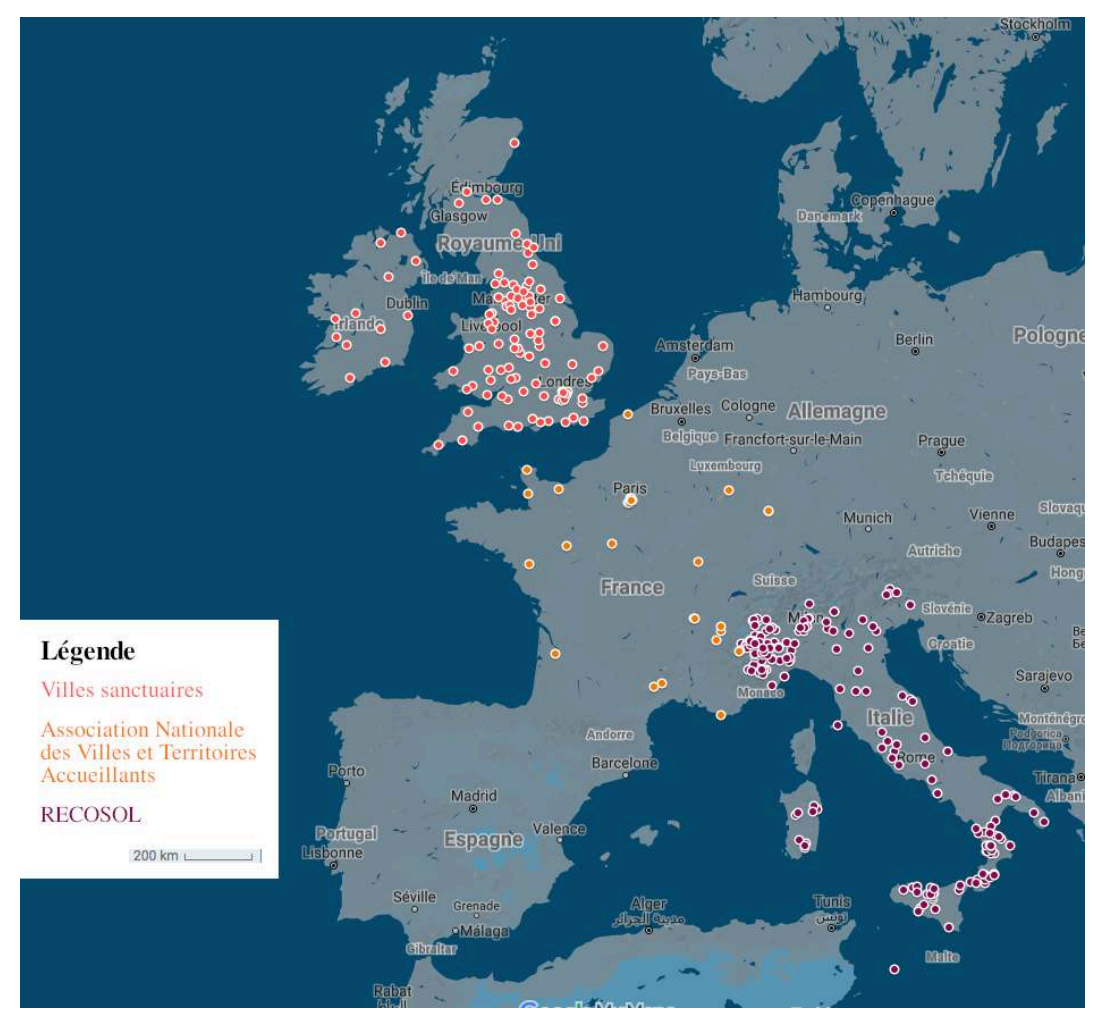

Conception et réalisation: Thomas Lacroix, 2019 / Google MyMaps

\section{Les réseaux municipaux transnationaux}

On retrouve, au niveau international, la même ligne de partage entre réseaux cooptés et / ou créés par les États et les organisations internationales d'un côté, et les réseaux spontanés de l'autre. Le paysage des réseaux transnationaux de villes est cependant extrêmement complexe, ce qui rend la distinction entre ces deux groupes plus incertaine.

\section{Les réseaux transnationaux de la para-diplomatie municipale}

Comme évoqué ci-dessus, les années 1990 ont été une période d'émergence de la «paradiplomatie » urbaine (c'est-à-dire les stratégies internationales mises en œuvre par les villes) (Viltard, 2008) au sein d'un espace politique européen. Le nombre de réseaux et de villes impliqués a continué à augmenter dans les années 2000. À partir de l'adoption de la directive « Race » en 2000, puis, en 2005, du Programme cadre pour la solidarité et la gestion des flux migratoires (COM (2005) 123 final), l'intégration devient l'axe principal de l'activisme urbain au niveau européen en matière migratoire. Les deux réseaux mentionnés ci-dessus, Eurocities et le CCRE, ont renforcé leur position aux côtés de la Commission européenne. Ils ont des positionnements différents dans le champ de cette para-diplomatie urbaine. Le CCRE est une organisation dont les origines remontent à l'après-guerre, mais qui a servi, depuis les années 1980, d'organe représentatif des collectivités territoriales auprès de la Commission. On peut la classer donc dans le groupe des organisations cooptées. Eurocités fut créé peu après, au début des années 1990. Comme nous l'avons vu ci-dessus, l'association est le résultat d'une 
mobilisation spontanée de grandes villes. Toutefois, Eurocités a acquis une légitimité qui en fait un partenaire essentiel de la Commission européenne. Elle s'est parfaitement insérée dans le tissu organisationnel européen et elle occupe aujourd'hui les mêmes bâtiments que le CCRE à Bruxelles. Leurs activités se ressemblent également. Ces deux réseaux sont engagés auprès de leurs membres afin de favoriser la collaboration interurbaine et de renforcer leurs capacités financières, réglementaires et institutionnelles, mais ils sont aussi actifs auprès des États et de la Commission européenne. Le CCRE, en particulier, rédige des amendements à la réglementation de l'UE afin de réaffirmer la place des autorités locales dans la gouvernance des migrations. Le pacte d'Amsterdam (2016) a facilité cette participation. Le document a élargi les capacités des villes à participer à la production de la législation de l'UE. L'intégration des migrants est l'une des priorités mentionnées dans le pacte.

Le vrai changement observé au cours de la dernière décennie, concerne l'émergence de réseaux mondiaux de la para-diplomatie municipale, incluant les autorités locales du Nord et du Sud. Là encore, ces réseaux peuvent être des rassemblements spontanées (tels que le Global Parliament of Mayors), ou encore des organisations représentatives pour des structures internationales. Le CGLU (Cités et Gouvernements Locaux Unis), créé en 2004, est, à ce titre, une institution clé puisqu'elle fait office de relai des municipalités auprès de l'ONU. Le CGLU prend la forme d'un " réseau de réseaux » qui s'appuie sur six relais continentaux (CGLU Afrique, Moyen Orient, Asie, Amérique du Nord, le CCRE et la FLACMA et le Mercociudades en Amérique latine), qui sont euxmêmes des structures fédérant les associations nationales. Cette architecture permet au CGLU d'avoir des ramifications auprès de l'ensemble des villes du monde.

Ces organisations sont à caractère généraliste, même si elles traitent toutes de la migration et de l'intégration dans des groupes de travail dédiés. Et ce ne sont pas des organisations à caractère idéologique créées en réaction aux politiques de migration des États : ces réseaux ont été formés pour favoriser l'institutionnalisation d'un espace diplomatique urbain pour les villes prêtes à se conformer à leur agenda international. Par conséquent, tous les membres ne sont pas investis sur les questions migratoires. Moins politisées, leur engagement se limite le plus souvent au partage de bonnes pratiques, le financement de projets ou encore de campagnes de sensibilisation.

\section{Les réseaux transnationaux de villes hospitalières}

Se sont également multipliées des organisations spécialement dédiées aux questions d'accueil et d'intégration. Certaines sont des regroupements de villes autour d'un projet militant. Le réseau ICORN (International Cities of Refuge Network) rassemble des villes qui accueillent des artistes issus de pays en conflit. Welcoming international est une structure fédérative qui fait le lien entre les associations Welcoming cities aux États-Unis, en Australie, en Nouvelle Zélande ou en Allemagne.

À côté de ces réseaux spontanés, on recense également un certain nombre d'organisations qui ont été créées par des organisations internationales sur des thématiques liées aux migrations: le réseau Intercultural Cities Network, etc. Par exemple, le réseau ICCAR (International Coalition of Inclusive and Sustainable Cities) au niveau international, est soutenu par l'UNESCO. Comme le CGLU, c'est un réseau de réseaux qui s'appuie sur des relais sur chaque continent, et notamment l'ECCAR (European Coalition if Cities against Racism) en Europe. Ces réseaux thématiques ont pour 
effet de diffuser des approches politiques, essentiellement dans le domaine de l'intégration et de la cohésion sociale.

Carte 2 : le réseau ICCAR

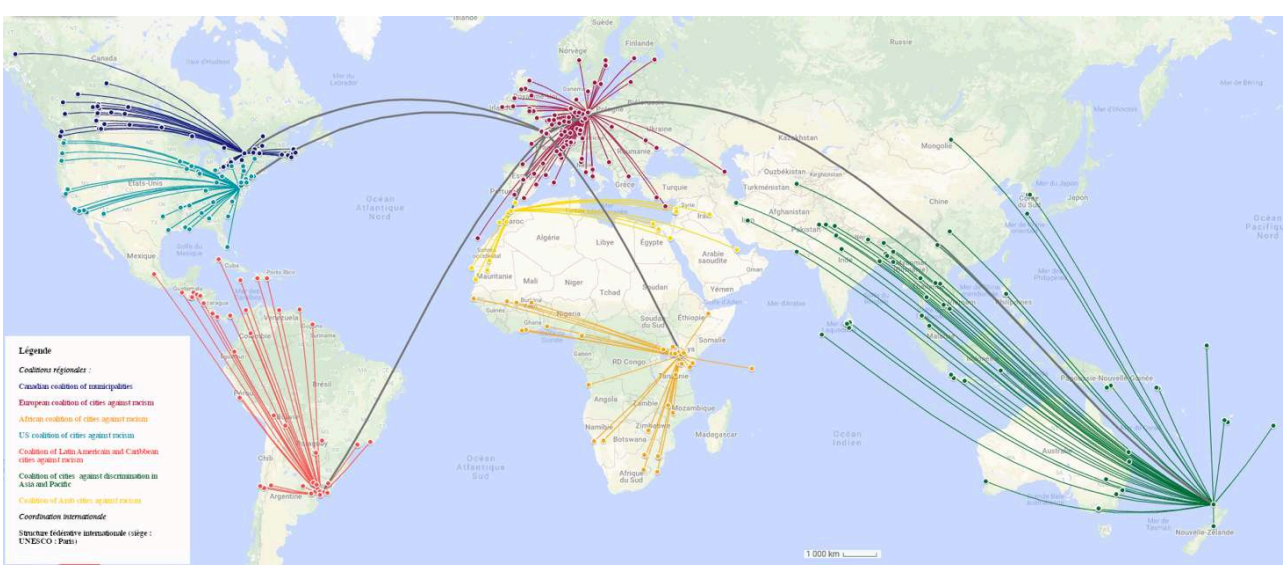

Conception et réalisation : Thomas Lacroix, 2019 / Google MyMaps

Parmi les réseaux initiés par les organisations internationales, le Forum des Maires pour la Mobilité humaine et le Développement tient une place spécifique. Il s'agit d'une institution créée par l'Organisation internationale pour les Migrations (OIM) et d'autres organisations internationales telles que le PNUD ou la Banque mondiale. En 2014, une première réunion a eu lieu à Barcelone, au cours de laquelle a été adopté «l'appel de Barcelone ", destiné à "mettre l'accent sur la nécessité d'amplifier la voix des villes dans les discussions mondiales sur les migrations ». En 2015, l'OIM organise une conférence internationale intitulée "Migration et villes » à Genève. Un rapport a suivi sur le rôle des villes dans la gestion de la migration. La même année, un deuxième forum a été organisé à Quito pour définir et présenter « l'agenda local en matière de migration et de développement ». Les troisième et quatrième édition du Forum ont été organisées en 2016 à Quezon City et à Berlin en 2017. La dernière a eu lieu à Marrakech en décembre 2018, parallèlement à la signature du Pacte mondial pour les migrations. Le Forum est devenu le troisième pilier du dispositif institutionnel de gouvernance internationale des migrations, aux côtés du Forum mondial sur la migration et le développement réunissant des représentants des États et du Forum social mondial réunissant des acteurs de la société civile. La création du Forum des maires fait partie intégrante d'une stratégie soutenue par des organisations internationales visant à mettre en place une gouvernance mixte des migrations internationales impliquant des acteurs publics (États et autorités locales) et privés (organisations de la société civile).

\section{Conclusion}

Cet article offre un aperçu de la diversité des réseaux de villes investis sur les questions migratoires et du virage global qui caractérise leur évolution récente. Il montre comment deux dynamiques convergentes ont structuré ce paysage : une dynamique ascendante de regroupements spontanés de villes autour d'un projet commun, une dynamique descendante de réseaux animés par des organisations internationales et leurs institutions représentatives. Dans ce paysage hétéroclite se croisent militants, fonctionnaires internationaux, élus, techniciens, etc. L'objectif de cet article a été de 
rendre compte de cette dynamique de mise en réseau. De prochaines recherches pourront donner corps à cette dynamique en portant la focale sur les acteurs de ces réseaux et la nature des rapports de force qui les sous-tendent: les maires, et autres agents municipaux, mais aussi des ONGs, des associations locales, des groupes d'habitants, voire des individus.

Nous avons tenté ici de mettre en évidence les facteurs qui expliquent cette dynamique. La « crise migratoire » a été un contexte qui a favorisé ce processus de mise en réseau, tant pour des villes à la recherche de soutiens techniques ou idéologiques que pour des organisations internationales œuvrant pour la mise en place d'une gouvernance mondiale des migrations. Mais, au-delà de ce contexte à court terme, j'ai mis en évidence deux "paradoxes » d'arrière-plan qui expliquent l'expansion des réseaux de villes liés à la migration. Le premier paradoxe est niché dans la vague de réformes de décentralisation qui a radicalement transformé la structure de la gestion économique au niveau local. Le deuxième paradoxe résulte d'une tension croissante entre les politiques d'intégration et les politiques migratoires. L'utilisation d'institutions et d'acteurs en contact avec les immigrés à des fins de contrôle, mais aussi la précarité croissante de la situation des immigrés ont conduit les autorités locales à prendre position sur des questions telles que les visas et l'asile. Elles induisent également un changement d'approche dans le traitement des populations immigrées. Un nouveau lexique de l'accueil semble se substituer au vocabulaire de l'intégration. Là encore, de futures recherches devront analyser l'émergence et la consistance politique qui se cache derrière cette terminologie.

Enfin, on voit aujourd'hui que l'OIM tente de canaliser ces différentes tendances au sein du Forum des Maires sur la Mobilité et le Développement. Il reste à voir si des principes d'action commune pourront unir des villes du Nord et du Sud, d'immigration et d'émigration. On verra alors si les villes peuvent réussir là où les États ont échoué.

\section{BIBLIOGRAPHIE}

Bulkeley, Harriet ; Davies, Anna ; Evans, Bob ; Gibbs, Gibbs ; Kern, Kristine ; Theobald, Kate (2003) Environmental governance and transnational municipal networks in Europe, Journal of Environmental Policy \& Planning, vol. 5, n 3 : pp. 235-254.

Flamant, Anouk (2014) Droit de cité! Construction et dilution d'une politique municipale d'intégration des étrangers dans les villes de Lyon, Nantes et Strasbourg (1981-2012), Lyon, Université de Lyon 2, $652 \mathrm{p}$.

Th. Doct. : Sc. Po : Lyon : 2014.

Florida, Richard (2014) The Rise of the Creative Class-Revisited : Revised and Expanded, New York: Basic Books, $512 \mathrm{p}$.

Fünfgeld, Hartmut (2015) Facilitating local climate change adaptation through transnational municipal networks, Current Opinion in Environmental Sustainability, n 12, pp. 67-73. 
Gazzotti, Lorena (2018) Local Governance, Civil Society and Migrants' Support to Local Development : Perspectives from Morocco, In Lacroix, T. ; Desille, A. (dir.) International Migrations and Local Governance, Basingstoke ; New York, Palgrave MacMillan, pp. 79-96 (Migration, Diasporas and Citizenship).

Hiam, Lucinda ; Steele, Sarah ; McKee, Martin (2018) Creating a 'hostile environment for migrants' : the British government's use of health service data to restrict immigration is a very bad idea. Health Economics, Policy and Law, vol. 13, n² 2, pp. 107-117.

Hoekstra, Myrte S. ; Kohlbacher, Josef ; Rauhut, Daniel (2018) Migration Governance in Three European Cities : New Local Paradigms ?, In Lacroix, T. ; Desille, A. (dir.) International Migrations and Local Governance, Basingstoke ; New York, Palgrave MacMillan, pp. 17-38 (Migration, Diasporas and Citizenship).

Hooghe, Liesbet (1995) Subnational mobilisation in the European Union, West European Politics, vol. 18, n 3, pp. 175-198.

International Organisation for Migration (2015) Migrants and Cities : New Partnerships to Manage Mobility, Geneva, International Organisation for Migration, 227 p. (World Migration Reort).

Ivanyna, Maksym ; Shah, Anwar (2012) How close is your government to its people? Worldwide indicators on localization and decentralization, Washington D.C., The World Bank, Policy research Working paper 6138.

Kern, Kristine ; Bulkeley, Harriet (2009) Cities, Europeanization and multi-level governance : governing climate change through transnational municipal networks, JCMS : Journal of Common Market Studies, vol. 47, n² 2, pp. 309-332.

Lacroix, Thomas (2018) Le Transnationalisme. Espace, Temps, Politique, Marne La Vallée, Université Paris Est, 166 p. (Habilitation à Diriger des Recherches)

HDR : Géo. : Marne La Vallée2014.

Lacroix, Thomas ; Desille, Amandine (dir.) (2018) International Migrations and Local Governance : A Global Perspective, Basingstoke ; New York, Palgrave MacMillan, 243 p. (Migration, Diasporas and Citizenship).

Manor, James (2004) User committees : a potentially damaging second wave of decentralisation?, The European Journal of Development Research, vol. 16, $\mathrm{n}^{\circ}$ 1, pp. 192-213.

Oomen, Barbara ; Baumgärtel, Moritz ; Durmus, Elif (2018) Transnational City Networks and Migration Policy. Utrecht University : Cities of refuge research [Disponible sur Internet].

Ridgley, Jennifer (2008) Cities of refuge : Immigration enforcement, police, and the insurgent genealogies of citizenship in US sanctuary cities, Urban Geography, vol. 29, n 1, pp. 53-77.

Saunier, Pierre-Yves ; Ewen, Shane (2008) Another global city. Historical Explorations into the Transnational Municipal Moment, 1850-2000, Basingstoke ; New York, Palgrave Macmillan, 242 p.

Solomos, John (2003) Race and racism in Britain, Basingstoke ; New York, Palgrave-Macmillan, 209 p.XTaylor, Peter J. ; Derudder, Ben (2004) World city network : a global urban analysis, London; New York, Routledge, $241 \mathrm{p}$.

Viltard, Yves (2008) Conceptualiser la « diplomatie des villes », Revue Française de science politique, vol. 58, $\mathrm{n}^{\circ} 3$, pp. 511-533.

World Economic Forum (2017) Migration and Its Impact on Cities, Genève, World Economic Forum, $172 \mathrm{p}$. [Disponible sur Internet]. 


\section{NOTES}

1. Arrondissements municipaux.

2. «Face aux flux migratoires, nous, les maires, sommes au pied du mur » Le Monde, 2017/12/16.

\section{RÉSUMÉS}

$\mathrm{Au}$ cours de la dernière décennie, le nombre de réseaux de villes créés pour résoudre les problèmes de migration n'a cessé de croître. En s'appuyant sur une base de données incluant 50 réseaux, cet article propose une définition de ce type d'organisation, donne la mesure de leur expansion et analyse les facteurs qui expliquent cette tendance. Ce phénomène s'inscrit dans l'évolution à long terme de la gouvernance locale: les municipalités se voient dotées de responsabilités plus importantes en matière de gestion des populations immigrées. Mais, plus récemment, la « crise migratoire » de 2015-2016 a incité les autorités locales à se mobiliser face à la sécurisation des politiques migratoires. Dans ce contexte, les organisations internationales s'appuient de plus en plus sur ces acteurs locaux pour construire une gouvernance migratoire « transnationale » où les États n'ont pas apporté de réponses adéquates.

\section{INDEX}

Mots-clés : espace urbain, ville, accueil, réseaux d'entraide, politique publique

Index géographique : Union européenne, Amérique du Nord

\section{AUTEUR}

\section{THOMAS LACROIX}

Directeur de recherche au CNRS, Maison Française d'Oxford

Chercheur associé à Migrinter (UMR 7301), au CERI (UMR 7050) et fellow de l'Institut

Convergence Migration

thomas.lacroix@univ-poitirs.fr 